\title{
Modular organization enhances the robustness of attractor network dynamics
}

\author{
Neeraj Pradhan ${ }^{1,2}$, Subinay Dasgupta ${ }^{3}$ and Sitabhra Sinha ${ }^{1}$ \\ ${ }^{1}$ The Institute of Mathematical Sciences, CIT Campus, Taramani, Chennai 600113, India. \\ ${ }^{2}$ Department of Physics, Birla Institute of Technology ES Science, Pilani 333031, India. \\ ${ }^{3}$ Department of Physics, University of Calcutta, 92 Acharya Prafulla Chandra Road, Kolkata 700009, India.
}

\begin{abstract}
Modular organization characterizes many complex networks occurring in nature, including the brain. In this paper we show that modular structure may be responsible for increasing the robustness of certain dynamical states of such systems. In a neural network model with threshold-activated binary elements, we observe that the basins of attractors, corresponding to patterns that have been embedded using a learning rule, occupy maximum volume in phase space at an optimal modularity. Simultaneously, the convergence time to these attractors decreases as a result of cooperative dynamics between the modules. The role of modularity in increasing global stability of certain desirable attractors of a system may provide a clue to its evolution and ubiquity in natural systems.
\end{abstract}

PACS numbers: 87.18.Sn,75.10.Nr,89.75.Fb,64.60.Cn

An ubiquitous property of complex systems is their modular organization [1], characterized by communities of densely connected elements with sparser connections between the different communities [2]. In the biological world, modules are seen to occur across many length scales, from the intra-cellular networks of protein-protein interactions [3, 4] and signaling pathways [5] to food webs comprising multiple species populations [6]. Although such groupings are primarily defined in terms of the structural features of the network topology, in several instances distinct modules have also been associated with specific functions. Indeed, in the case of the brain, modular organization at the anatomical level has long been thought to be paralleled at the functional level of cognition [7]. By observing the effects of isolating or disconnecting different brain areas on the behavior of subjects, the functional specialization of spatially distinct modules have been established at different length scales [8] - from hemispheric specialization to minicolumns comprising a few hundred cells which have been proposed as the basic information processing units of the cerebral cortex $[9,10]$. More recently, the analysis of neurobiological data using graph theoretic techniques [11] has further established the modular nature of inter-connections between different areas of the mammalian cortex. The structural modules revealed by tracing the anatomical connections in mammalian brains [12, 13] are complemented by the observation of functionally defined networks having modular character [14, 15]. Such functional networks have been reconstructed from MRI and fMRI experiments on both human [16] and non-human [17] subjects, by considering two brain areas to be connected if they are simultaneously active when the subject performs a specific behavioral task.

The wide-spread occurrence of modularity prompts the question as to why this structural organization is so ubiquitous [18]. One possible reason is that it enhances communication efficiency by decreasing the average network path length while allowing high clustering to help localize signals within subnetworks [19]. However, of more interest is the possibility that modularity may play a crucial role in the principal function of the system, viz., information processing in the case of brain networks. This possibility has been investigated in detail for the somatic nervous system of the nematode C. elegans [20]. It is therefore intriguing to speculate whether modularity is responsible for efficient information processing in brains of more evolved organisms, the mammalian cortex in particular. To explore this idea further we can study the effect of modular structure on the dynamics of attractor network models with threshold-activated nodes, which exhibit multiple stable states or "memories" [21, 22]. These models were originally developed to understand how the nervous system communicates among its component parts and learns associations between different stimuli so that a memorized pattern can be retrieved in its entirety from a small part or a noise-corrupted version of it given as input ("associative memory"). Indeed, recent experiments indicate that the spatiotemporal activation dynamics in neocortical networks converge to one of several different persistent, stable patterns which resemble the behavior observed in such models [23]. However, the properties of attractor networks are of more general interest and have been used to understand systems outside the domain of neurobiology, as for example, the network involved in intracellular signaling where communication between molecules within a cell take place through multiple interacting pathways [24, 25]. In the attractor networks, desired patterns are stored by using a learning rule to determine the connection weights between the nodes. This ensures that the update (or recall) dynamics of the network makes it converge to these pre-specified dynamical states when an input initial state of the system is transformed into an output state defined over the same set of nodes by the collective dynamics of the network. Using such simplified models have the advantage of making the observed phenomena simpler to analyze and also to obtain results that are independent of specific bio- 
logical details of different types of neurons and synaptic connections.

In this paper we show that if we want to store $p$ (say) patterns in a network with a given number of nodes and links, then the convergence to an attractor corresponding to any of the stored patterns (i.e., recall) will be most efficient when the network has an optimal modular structure, provided the number of patterns is not too large $\left(p<p_{\max }\right)$. If the degree of modularity is increased or decreased from the optimum value, the reliability with which the patterns are recalled decreases. This optimal efficiency of recall originates from the network dynamics itself. Some of the modules converge quickly to attractors corresponding to parts of stored patterns and then help other modules to reach the attractor corresponding to the entire stored pattern through interactions via intermodular links. If the modularity is increased (i.e., if the number of intra-modular links is increased while reducing the number of inter-modular links to keep the average degree fixed), the modules cannot interact with each other strongly enough due to fewer number of intermodular links and the performance of the network is less efficient. On the other hand, if the modularity is decreased, the modules themselves become sparsely connected and cannot reach an attractor rapidly. Also, if we try to store a larger number of patterns $\left(p \geq p_{\max }\right)$, the advantage of modularity disappears because of the generation of a large number of spin-glass states which correspond to spurious patterns.

The attractor network model we have used to investigate the role of modularity is constructed such that the $N$ nodes comprising it are divided into $n_{m}$ modules, each having $n\left(=N / n_{m}\right)$ nodes [19]. The connection probability between a pair of nodes belonging to the same module is $\rho_{i}$, while that between nodes belonging to different modules is $\rho_{o}$. The modular nature of the network can be varied continuously by altering the ratio of inter- to intra-modular connectivity, $r=\frac{\rho_{o}}{\rho_{i}} \in[0,1]$, keeping the average degree $\langle k\rangle$ fixed (Fig. 11). For $r=0$, the network is fragmented into $n_{m}$ isolated clusters, whereas at $r=1$, it is a homogeneous or Erdos-Renyi random network. We ensure that the resulting adjacency matrix $\mathbf{A}$ (i.e., $A_{i j}=1$ if $i, j$ are connected, and 0 , otherwise) is symmetric. We have explicitly verified that the results reported below do not change appreciably if $\mathbf{A}$ is nonsymmetric (corresponding to a directed network).

The time-evolution of the system is governed by the dynamics of the variables associated with each node of the network. An Ising spin $\sigma_{i}= \pm 1$ is placed at each node which may represent any binary state variable, such as a two-state neuron (firing $=1$, inactive $=-1$ ). The state of the spins are evaluated at discrete time-steps using random sequential updating according to the following deterministic (or zero temperature) dynamics:

$$
\sigma_{i}(t+1)=\operatorname{sign}\left(\Sigma_{j} A_{i j} W_{i j} \sigma_{j}(t)\right)
$$

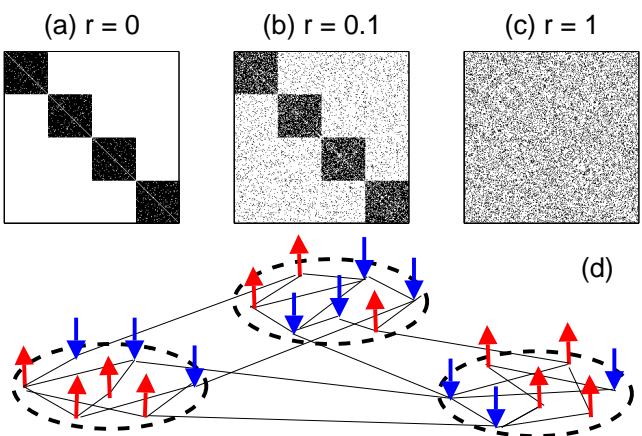

FIG. 1: (a-c) Adjacency matrices A defining the network connections at different values of the modularity parameter $r$ for $N=256$ nodes arranged into $n_{m}=4$ modules (average degree $\langle k\rangle=60)$. Starting from a system of isolated clusters (a, $r=0$ ), by increasing $r$ we obtain modular networks (b, $r=0.1$ ) eventually arriving at a homogeneous network (c, $r=1$ ). The connection structure of modular networks in the intermediate range $0<r<1$ is shown schematically in (d). The connection weights have different magnitudes and signs.

where, $W_{i j}$ is the connection strength between neurons $i$ and $j$. The function $\operatorname{sign}(z)=1$, if $z>0,=-1$, if $z<0$ and randomly chosen to be \pm 1 if $z=0$. The weight associated with each link is evaluated using the Hebbian learning rule 22] for storing $p$ random patterns in an associative network:

$$
W_{i j}=\frac{1}{\langle k\rangle} \Sigma_{\mu} \xi_{i}^{\mu} \xi_{j}^{\mu}, W_{i i}=0,
$$

$\xi_{i}^{\mu}$ being the $i$-th component of the $\mu$-th pattern vector $(\mu=1, \ldots p)$. Each of the stored patterns are generated randomly by choosing each component to be +1 or -1 with equal probability. Starting from an arbitrary initial state, the network eventually converges to a timeinvariant stable state or attractor. The overlap of an attractor of the network dynamics $S^{*}=\left\{\sigma_{i}^{*}\right\}$ with any of the stored patterns can be measured as $m_{\mu}=\frac{1}{N} \Sigma_{i} \sigma_{i}^{*} \xi_{i}^{\mu}$. As we are interested in the set of all the attractors of stored patterns rather than one specific pattern, we focus our attention on the maximum overlap with the stored patterns, $m=\max _{\mu}\left|m_{\mu}\right|$. To examine the global stability of the attractors corresponding to the stored patterns, we use random strings as the initial state of the network which should have almost no overlap with any of the stored patterns, on average. The probability $v_{g} \equiv\left\langle\operatorname{Prob}\left(m>m_{o}\right)\right\rangle$ that such a random initial state eventually almost converges to one of the stored patterns, gives an estimate of the overall volume that the basins of attraction of stored patterns occupy in the $N$-dimensional network configuration space $\{S\}$. Here $m_{o}$ is a threshold for the overlap of the asymptotic stable state above which the network can be considered to have recalled a pattern successfully and $\langle\ldots\rangle$ indicates averaging over many different network configurations $\mathbf{A}$, 

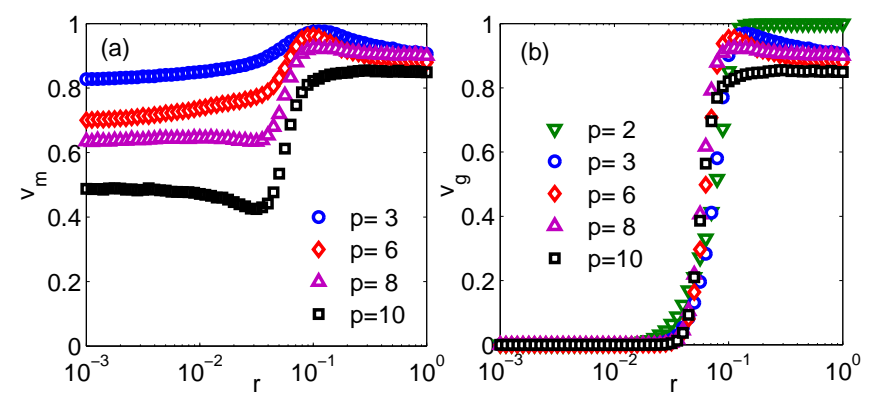

FIG. 2: Fractional volume of phase space occupied by the basins of attraction of the stored patterns in a single module $\left(v_{m}\right)$ and the entire random modular network $\left(v_{g}\right)$. Note that, when the number of stored patterns is within a critical range $\left(p_{\min }=2<p<p_{\max }=9\right)$, these quantities show a non-monotonic variation with $r$, having a peak around $r_{c} \simeq 1 /\left(n_{m}-1\right) \sim 0.14$. Results are shown for $N=1024$, $n_{m}=8$ and $\langle k\rangle=120$. Different numbers of stored patterns $p$ are indicated using various symbols.

as well as, pattern ensembles $\{\xi\}$ and initial states. The value of the threshold $m_{o}$ has been taken to be 0.95 for most of the analysis presented here; we have verified that varying it over a small range does not alter our results. In a similar way, we can define overlap for each module, $m_{\mu}(\alpha)=\frac{1}{n} \Sigma_{i} \sigma_{i}^{*}(\alpha) \xi_{i}^{\mu}(\alpha)$ where the sum is over all spins in the $\alpha$-th module with $\alpha=1, \ldots, n_{m}$ being an index running over the different modules. The relative size of the basins of attraction at the modular scale is characterized by the quantity $v_{m}=\left\langle\left\langle\operatorname{Prob}\left(m(\alpha)>m_{o}\right)\right\rangle_{\alpha}\right\rangle$, where $m(\alpha)=\max _{\mu}\left|m_{\mu}(\alpha)\right|$ and $\langle\ldots\rangle_{\alpha}$ indicates averaging over all the modules.

We first look at how the total volume of the configuration space occupied by the basins of attraction for stored patterns $\xi^{\mu}$ changes as the modular character of the network is altered by varying $r$ for a fixed $\langle k\rangle$. Fig. 2 shows the combined fractional volume of the phase space occupied by the basins of attraction of the stored patterns for the entire network $\left(v_{g}\right)$ as well as for the corresponding sub-patterns in a single module $\left(v_{m}\right)$. Different curves indicate various number of stored patterns $p$. We immediately notice that while $v_{m}$ has finite values over the entire range of $r, v_{g}$ is zero at low values of $r$ where a module is connected to the rest of the network by very few links, if at all. The value of $r$ at which $v_{g}$ starts rising from 0 appears to be independent of the number of stored patterns $p$. Below this value of $r$, the connectivity between the modules is insufficient to recall the entire stored pattern, even though individual modules may have complete overlap with different stored patterns. To explain the situation, we can decompose each stored pattern in terms of $n_{m}$ sub-patterns defined over the different modules, viz., $\xi^{\mu}=\left\{\xi^{\mu}(\alpha)\right\}$, where $\alpha=1, \ldots, n_{m}$. Starting from a random initial state, a module $\alpha$ may converge to an attractor corresponding to any of the $n_{m}$ different subpat- terns $\xi^{\mu}(\alpha)$. As the recall dynamics within each module is nearly independent of the other modules for low $r$, they may each converge to sub-parts of different patterns, i.e., the value of $\mu$ would not be identical for the attractors of all the $n_{m}$ modules. Thus, the resulting attractor for the entire network corresponds to a "chimera" memory state, $\left\{\xi^{\mu_{1}}(1), \ldots, \xi^{\mu_{n_{m}}}\left(n_{m}\right)\right\}$, i.e., a spurious pattern comprising fragments of different stored patterns [26, 27].

From the perspective of enhanced robustness of the dynamical attractors of the entire network, even more interesting is the behavior of $v_{g}$ and $v_{m}$ when $r$ is increased further after the modules have become interconnected appreciably. Over an intermediate range of $p_{\min }<p<p_{\max }$, we notice a non-monotonic variation of both $v_{g}$ and $v_{m}$ with respect to $r$. Fig. 2 2 shows that both curves attain a maximum around $r_{c} \sim \frac{n-1}{N-n} \simeq \frac{1}{n_{m}-1}$, where a neuron has the same number of connections with nodes belonging to its own module as it has with neurons belonging to different modules. When the relative number of inter-modular connections are increased beyond $r_{c}$, the fractional volume of configuration space occupied by the attractors corresponding to the stored patterns tend to decrease. This implies that the homogeneous network $(r=1)$ is actually less robust than its modular counterpart $\left(r \simeq r_{c}\right)$ in terms of global stability of the stored attractors. As $p$ increases beyond $p_{\max }$, both $v_{g}$ and $v_{m}$ decrease at the resulting high loading fraction $p /\langle k\rangle$ through the generation of a large number of spin-glass states [22]. We have explicitly verified that the maximum number of stored patterns $p_{\max }$ beyond which the non-monotonic nature of the variation is lost, increases when the total number of neurons $N$ is increased, keeping the overall density of connections, $\langle k\rangle /(N-1)$, and the number of modules, $n_{m}$, fixed [28].

For low values of $p$, i.e., $p \leq p_{\text {min }}$, both $v_{g}$ and $v_{m}$ increase with $r$ eventually reaching 1 and becoming independent of $r$ once the connectivity between the modules become appreciable. We find from our numerical results that $p_{\min }=2$, independent of the system size $N$ or other model parameters. This observation helps in identifying the key mechanism for the non-monotonic variation of $v_{g}$ with $r$. While at low $r, v_{g}$ is small because the low connectivity among modules favor the chimera states, at very large $r$ the attractors corresponding to the stored patterns have to compete with mixed states. Mixed states are spurious attractors that correspond to symmetric combinations of an odd number of stored patterns (e.g., $\xi^{1}+\xi^{2}+\xi^{3}$ ) which exist for all $p>2$. This is explicitly shown by the distribution of the overlap, $m$, of the attractors of a network with any of the $p$ stored patterns (shown in Fig. 3 for $p=4$ ). For low values of $r$, the dominance of chimera states result in low overlap values. When the modules become highly inter-connected as $r \rightarrow 1$, most randomly chosen initial strings will converge to a stored pattern resulting in a large peak at $m=1$ in the overlap distribution. However, we also no- 

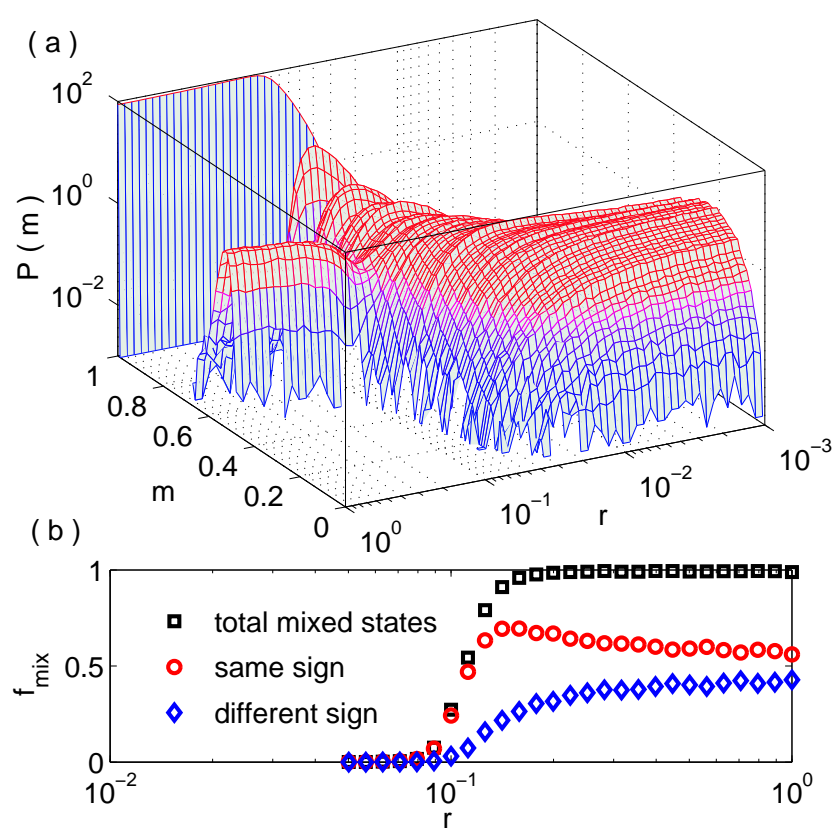

FIG. 3: (a) Distribution of the overlap of the attractors of the network dynamics with the stored patterns in a random modular network, at different values of the modularity parameter $r . P(m)$ is the probability of having overlap $m$. Complete overlap with the stored patterns $(m=1)$ becomes more probable as $r$ becomes larger than a threshold value. However, at large values of $r$, there is a secondary peak around $m_{g} \sim 0.5$ corresponding to mixed states (i.e., linear combination of odd number of stored patterns). This peak shows a dip at $r_{c} \simeq 1 /\left(n_{m}-1\right) \sim 0.14$. (b) The variation, as a function of $r$, of the fraction of total number of spurious attractors that are mixed states, $f_{m i x}$. For $r>r_{c}$, the mixed states account for almost all the attractors not corresponding to any of the stored patterns. They can be either combinations having the same sign (square) or different signs (diamond). Results shown for $N=1024, n_{m}=8,\langle k\rangle=120$ and number of stored patterns, $p=4$.

tice a smaller peak around $m \simeq 0.5$, which corresponds to 3 -pattern mixed states (which have overlap of 0.5 with each of the three constituent stored patterns). Note that as $r$ is gradually decreased from 1 , about $r \simeq r_{c}$ the $m$ distribution shows a sharp dip for overlaps around 0.5. This corresponds to an increase in the phase space volume occupied by the attractors of the stored patterns at the expense of the mixed states. A similar dip in the distribution is also observed for the corresponding overlap around 0.5 for each module (figure not shown). Thus, the cooperative interactions between the different modules not only affect the recall dynamics at the global level, but also locally within each module.

Fig. 3 (b) shows explicitly that the attractors not corresponding to any of the stored patterns, belong almost exclusively to mixed states at high $r$. In principle, these combinations can be of same sign (e.g., $\xi^{1}+\xi^{2}+\xi^{3}$ ) or

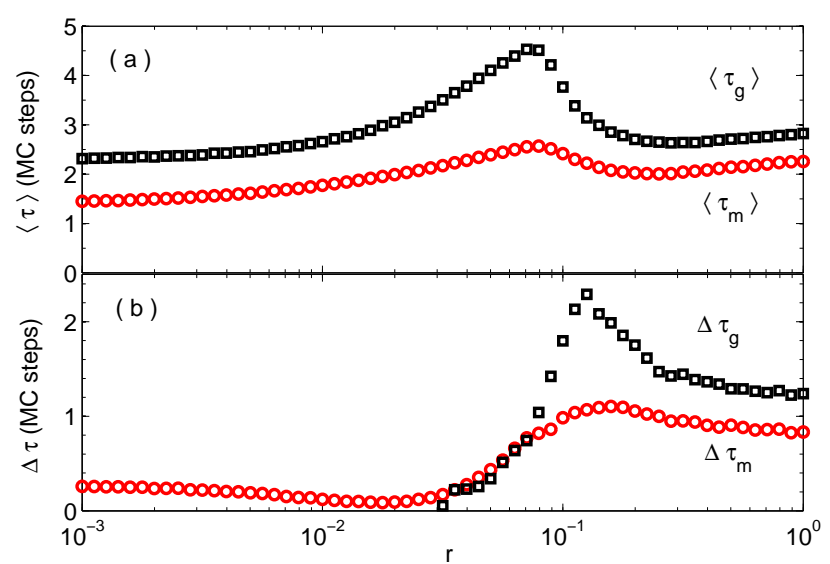

FIG. 4: (a) The average convergence time $\tau$ to different attractors in a random modular network, shown for individual modules $\left(\left\langle\tau_{m}\right\rangle\right.$, circles $)$ and the entire network $\left(\left\langle\tau_{g}\right\rangle\right.$, squares). It is measured in terms of Monte Carlo (MC) steps required to reach a time-invariant state starting from a random initial configuration. (b) The difference in the average convergence times (in MC steps) to an attractor not corresponding to a stored pattern $(m<0.95)$ and to one of the stored patterns, $\Delta \tau$. The difference is shown for both an individual module $\left(\Delta \tau_{m}\right.$, circles $)$ and the entire network $\left(\Delta \tau_{g}\right.$, squares $)$. The peak close to $r_{c} \sim 0.14$ corresponds to a significantly faster convergence to the stored patterns relative to the other attractors. Results shown for $N=1024, n_{m}=8,\langle k\rangle=120$ and $p=4$.

different signs (e.g., $\xi^{1}-\xi^{2}+\xi^{3}$ ). The curves corresponding to each of these show that although the latter has a higher number of possible combinations, it is the attractors corresponding to the same sign combinations which occupy a larger portion of the phase space. This is a consequence of the Hebbian learning rule, which provides a bias for the same sign combinations in preference to the different sign combinations.

So far we have discussed the long-time asymptotic properties of the system. The dynamical aspect represented by the time required to reach equilibrium also exhibits unexpected properties. Fig. 4 shows that the network converges faster to attractors corresponding to stored patterns as compared to mixed states (and other attractors that do not have significant overlap with any of the stored patterns), at both the modular and the network level. Moreover, this difference is slightly enhanced close to $r_{c}$, the modular configuration where the basins of the stored patterns cover the largest fraction of the configuration space. The non-monotonic variation of the convergence time with decreasing modularity arises as a result of two competing effects: increasing $r$ decreases the intra-modular connectivity, resulting in increasing time for each module to relax to an attractor; on the other hand, this is accompanied by an increase in the connections between modules, that eventually causes 
the entire system to relax faster to attractors. This dynamical picture provides us with a possible clue as to the enhanced global stability of the attractors corresponding to stored patterns close to $r_{c}$. As there is a distinct time-scale separation between the convergence dynamics at the modular (or local) and at the global scale for such networks [19, 29], the state of a specific module may evolve to reach a sub-pattern corresponding to a part of one of the stored patterns much faster than the network can converge to an attractor. Once this happens, this module biases the convergence of the other modules connected to it (via Hebbian inter-modular links) to the pattern to which it has converged. This increases the likelihood of convergence of the entire network to a particular pattern through cooperative behavior among the modules, something that is absent when the modules are disconnected or the network is homogeneous.

In this paper we have shown that modular organization in the connection structure of a network of threshold-activated elements can result in increased robustness of dynamical attractors associated with certain pre-specified states. These states may represent solutions to computational tasks or implement memorized patterns of activity. The modularity of the network allows these states to cover the maximum volume of its phase space with their basins, an outcome of cooperative behavior between the convergence or recall dynamics in the different modules. Our results have special relevance to the question of how cognitive states arise from interactions between a large number of brain regions, each comprising many neurons. Neurobiological evidence exists that cortical activity consists of rapid integration of signals across brain regions that are in spatially distinct locations and which occurs in a self-organized manner through interactions between the elements of the network of brain areas [30]. The empirical observation of modular cortical organization and the occurrence of distinct, persistent activity patterns corresponding to attractor dynamics raises the intriguing possibility that evolution may have selected modularity because of the robustness it imparts to the underlying system. Future extensions of the work reported here may involve considering the effect of noise, i.e., investigating the recall dynamics at a finite temperature. Another possibility is to investigate the role of hierarchical arrangement of modules that have recently been reported in different biological systems [31, 32], including the brain [33, 34]. Our results may also potentially be used to understand why attractor networks with smallworld connection topology show a small increase in global stability relative to random networks, although the local stability of stored patterns are unaffected [35 38$]$.

We would like to thank R. K. Pan for helpful discussions. This work is supported in part by CSIR, UGCUPE, IMSc Associates program and IMSc Complex Sys- tems (XI Plan) Project.

[1] L. H. Hartwell, J. J. Hopfield, S. Leibler and A. W. Murray, Nature (Lond.) 402, C47 (1999).

[2] M. Girvan and M. E. J. Newman, Proc. Natl. Acad. Sci. USA 99, 7821 (2002).

[3] B. Schwikowski, P. Uetz and S. Fields, Nature Biotech. 18, 1257 (2000).

[4] A. W. Rives and T. Galitski, Proc. Natl. Acad. Sci. USA 100, 1128 (2003).

[5] D. A. Lauffenburger, Proc. Natl. Acad. Sci. USA 97, 5031 (2000).

[6] A. E. Krause, K. A. Frank, D. M. Mason, R. U. Ulanowicz and W. W. Taylor, Nature (Lond.) 426, 282 (2003).

[7] J. A. Fodor, The Modularity of Mind, MIT Press, Cambridge, Mass., 1983.

[8] M. S. Gazzaniga, Science 245, 947 (1989).

[9] V. B. Mountcastle, in: The Mindful Brain (Eds. G. M. Edelman and V. B. Mountcastle), MIT Press, Cambridge, Mass., 1983.

[10] D. P. Buxhoeveden and M. F. Casanova, Brain 125, 935 (2002).

[11] E. Bullmore and O. Sporns, Nature Rev. Neurosci. 10, 186 (2009).

[12] C.-C. Hilgetag, G. A. P. C. Burns, M. A. O'Neill, J. W. Scannell and M. P. Young, Phil. Trans. R. Soc. Lond. B 355, 91 (2000).

[13] Z. J. Chen, Y. He, P. Rosa-Neto, J. Germann, J. and A. C. Evans, Cereb. Cortex 18, 2374 (2008).

[14] S. Achard, R. Salvador, B. Whitcher, J. Suckling, and E. Bullmore, J. Neurosci. 2663 (2006).

[15] D. Meunier et al, Neuroimage 44, 715 (2009).

[16] R. Salvador, J. Suckling, M. Coleman, J. Pickard, D. Menon and E. Bullmore, Cereb. Cortex 151332 (2005).

[17] A. J. Schwarz, A. Gozzi and A. Bifone, Magn. Reson. Imaging 26, 914 (2008)

[18] R. K. Pan and S. Sinha, Phys. Rev. E 76, 045103(R) (2007).

[19] R. K. Pan and S. Sinha, EPL 8568006 (2009)

[20] R. K. Pan, N. Chatterjee and S. Sinha, PLoS One 5, e9240 (2010)

[21] J. J. Hopfield, Proc. Natl. Acad. Sci. USA 79, 2554 (1982).

[22] D. J. Amit, Modeling Brain Function (Cambridge Univ. Press, Cambridge, 1989)

[23] R. Cossart, D. Aronov and R. Yuste, Nature (Lond.) 423, 283 (2003).

[24] D. Bray, Nature (Lond.) 376307 (1995).

[25] D. Bray, Science 3011864 (2003).

[26] D. O. O'Kane and A. Treves, J. Phys. A 255055 (1992)

[27] D. O. O'Kane and D. Sherrington, J. Phys. A 262333 (1993)

[28] For example, $p_{\max }$ increases from 6 for $N=512$ to 9 for $N=1024$ and 12 for $N=2048$ when $\langle k\rangle /(N-1) \simeq 0.117$ and $n_{m}=8$.

[29] S. Dasgupta, R. K. Pan and S. Sinha, Phys. Rev. E 80, 025101(R) (2009)

[30] O. Sporns and C. J. Honey, Proc. Natl. Acad. Sci. USA 10319219 (2006)

[31] E. Ravasz et al, Science 297, 1551 (2002). 
[32] R. K. Pan and S. Sinha, Pramana 71, 331 (2008).

[33] L. Ferrarini et al, Human Brain Mapping 30, 2220 (2008).

[34] D. Meunier et al, Frontiers in Neuroinformatics 3, 37 (2009).

[35] L. G. Morelli, G. Abramson and M. N. Kuperman, Eur. Phys. J. B 38495 (2004).
[36] P. N. McGraw and M. Menzinger, Phys. Rev. E 68 047102 (2003).

[37] B. J. Kim, Phys. Rev. E 69 045101(R) (2004).

[38] H. Oshima and T. Odagaki, Phys. Rev. E 76036114 (2007). 